\title{
Evolution of the protease-activated receptor family in vertebrates
}

\author{
MIN JIN $^{1-3 *}$, HAI-WEI YANG ${ }^{4 *}$, AI-LIN TAO ${ }^{1}$ and JI-FU WEI ${ }^{1,2}$ \\ ${ }^{1}$ Guangdong Provincial Key Laboratory of Allergy and Clinical Immunology, \\ The State Key Laboratory of Respiratory Disease, The Second Affiliated Hospital of Guangzhou Medical University, \\ Guangzhou, Guangdong 510260; ${ }^{2}$ Research Division of Clinical Pharmacology, The First Affiliated Hospital \\ of Nanjing Medical University, Nanjing, Jiangsu 210029; ${ }^{3}$ School of Life Science and Technology, \\ China Pharmaceutical University, Nanjing, Jiangsu 210009; ${ }^{4}$ Department of Urology, \\ The First Affiliated Hospital of Nanjing Medical University, Nanjing, Jiangsu 210029, P.R. China
}

Received May 18, 2015; Accepted January 13, 2016

DOI: $10.3892 / \mathrm{ijmm} .2016 .2464$

\begin{abstract}
Belonging to the $\mathrm{G}$ protein-coupled receptor (GPcr) family, the protease-activated receptors (Pars) consist of 4 members, PAR1-4. PARs mediate the activation of cells via thrombin, serine and other proteases. Such protease-triggered signaling events are thought to be critical for hemostasis, thrombosis and other normal pathological processes. In the present study, we examined the evolution of PARs by analyzing phylogenetic trees, chromosome location, selective pressure and functional divergence based on the 169 functional gene alignment sequences from 57 vertebrate gene sequences. We found that the 4 PARs originated from 4 invertebrate ancestors by phylogenetic trees analysis. The selective pressure results revealed that only PAR1 appeared by positive selection during its evolution, while the other PAR members did not. In addition, we noticed that although these PARs evolved separately, the results of functional divergence indicated that their evolutional rates were similar and their functions did not significantly diverge. The findings of our study provide valuable insight into the evolutionary history of the vertebrate PAR family.
\end{abstract}

Correspondence to: Professor Ai-Lin Tao, Guangdong Provincial Key Laboratory of Allergy and Clinical Immunology, The State Key Laboratory of Respiratory Disease, The Second Affiliated Hospital of Guangzhou Medical University, 250 Changgang East Road, Guangzhou, Guangdong 510260, P.R. China

E-mail: taoailin@gzhmu.edu.cn

Professor Ji-Fu Wei, Research Division of Clinical Pharmacology, The First Affiliated Hospital of Nanjing Medical University, 300 Guangzhou Road, Nanjing, Jiangsu 210029, P.R. China

E-mail: weijifu@hotmail.com

*Contributed equally

Key words: protease-activated receptors, phylogenetic analysis, positive selection, functional divergence

\section{Introduction}

Discovered in the 1990s, protease-activated receptors (PARs) are membrane-spanning proteins that belong to the G proteincoupled receptor (GPCR) family and exist on the surface of cells in a wide variety of tissues (1). The PARs contain 7 transmembrane (TM1-7) helices, an extracellular amino terminal domain, 3 intracellular loops (ICL1-3), 3 extracellular loops (ECL1-3) and an intracellular carboxyl terminal domain. As well as connecting TM4 and TM5, ECL2 also makes a disulfide bond with TM3 that is conserved amongst GPCRs and contributes to receptor or structural stability (2). The PAR family consists of 4 members, PAR1-4. PAR1, PAR3 and PAR4 are thrombin receptors, while PAR2 is a receptor for serine proteases (including trypsin, mast cell tryptase, as well as factors Xa and VIIa, etc.) $(1,3,4)$.

Human PAR1 has been identified as a protein containing 425 residues, including an amino-terminal signal sequence of 20 residues and an extracellular amino-terminal domain of 75 residues (5). PAR1 is activated when cleaved by thrombin at a site between Arg41 and Ser42, exposing a new N-terminal tethered ligand domain with the sequence SFLLRN. The new $\mathrm{N}$-terminus acts as a tethered ligand and binds intramolecularly to the body of the receptor to effect TM signaling $(6,7)$.

Human PAR 2 consists of 397 amino acids with a molecular weight of $44 \mathrm{kDa}$. It has an extracellular N-terminal domain of 75 amino acids tethered to a TM domain of 155 amino acids, assembled in 7 pseudo-parallel helical sequences connected by 3 extracellular and 3 intracellular loops of 117 amino acids, with a small eighth helix within the intracellular C-terminus of 50 amino acids. The extracellular N-terminus of PAR2 is cleaved at a site between $\mathrm{Arg} 47$ and Gly48 by protease (mainly serine protease), and the newly exposed $\mathrm{N}$-terminus is GYPGQV. The exposed $\mathrm{N}$-terminus, known as a tethered ligand, then folds back and self-activates PAR2 through binding to conserved regions of ECL2 and/or the PAR2 TM region (1,8-10).

PAR3 is a typical GPCR with a thrombin cleavage site between Lys38 and Thr39, which has $27 \%$ amino acid sequence similarity to PAR1 and 28\% similarity to PAR2 (11). After being cleaved by thrombin, PAR3 exposes a new amino terminus (TFRGAP) which interacts with the receptor as a tethered ligand (12). 
Human PAR4 is a 385-amino acid protein with a potential thrombin cleavage site in the extracellular amino-terminal domain between Arg47 and Gly48, and has 33\% similarity to the other human PARs (13). PAR4 is one of the thrombin receptors on human platelets and a potential target for the management of thrombotic disorders. Briefly, when these receptors are activated by proteinases at the specific cleavage site, the extracellular $\mathrm{N}$-terminus of the receptor, a new $\mathrm{N}$-terminus, is exposed. The new $\mathrm{N}$-terminus has been found to act as a tethered ligand and binds intramolecularly to initiate cellular signals (13).

It has been demonstrated that following activation, the PAR family is able to stimulate complex intracellular signaling networks through classical $G$ protein-mediated pathways and $\beta$-arrestin signaling pathways (14). The signaling pathways induced by the activation of PARs play a key role in a number of physiological and pathological processes. For example, it has been demonstrated that PARs expressed on cells are involved in immune responses and inflammation, regulate endothelial-leukocyte interactions and modulate the secretion of inflammatory mediators or neuropeptides (4). PARs expressed on platelets and the vascular endothelium play important roles in normal blood vessel biology, which contribute to the pathogenesis of several cardiovascular diseases, including atherosclerosis, restenosis and thrombosis (15). Thus, it has been suggested that the PARs are potential therapeutic targets for the treatment of inflammation, hemostasis, thrombosis, vascular dysfunction and cancer (16-19). Apart from studies on the structure and function of PARs, few studies to date have focused on the genetic evolution of the family of PARs. Kahn et al compared the amino acid sequences, gene structures, locus organization and chromosomal locations in human and mouse PARs (20). Xu et al found that the function of PARs was conserved among vertebrates, after analyzing the expression patterns in zebrafish and their mammalian human and mouse counterparts (19). Thus far, however, to the best of our knowleged, there are no studies available on the global evolution of the PAR family in vertebrates. In this study, we describe the evolutionary genetic association between the members of the PAR family based on the analysis of phylogenetic trees, chromosome location, selective pressure and functional divergence.

\section{Materials and methods}

Sequence data collection. PAR gene sequences were obtained, based on their orthologous and paralogous relationship, by querying the Ensembl genome assemblies (http://www.ensembl. org/index.html) using the human PAR gene sequences. The obtained PAR sequences were then used as queries to obtain the best hit in BLAST at the NCBI database (http://www.ncbi. nlm.nih.gov/pubmed).

Multiple alignment and phylogenetic analysis. The protein coding sequences of the PAR gene family were aligned using the ClustalW program in MEGA 5.1 (21). The aligned sequences were used for the subsequent phylogenetic analysis. MEGA 5.1 was used to construct a maximum likelihood (ML) tree of the PAR family with the best fitting model of JTI+G+F that was selected by a model test in the same package with a bootstrap value based on 1,000 repetitions. MrBayes v3.1.2 was used to construct a Bayesian inference tree with the nucleotide substitution model (22). The Markov chain Monte Carlo (MCMC) technique was used for the PAR family. The repetitions run for PAR1, PAR2, PAR3 and PAR4 were 2,000,000, 3,000,000, 1,000,000 and 1,000,000 generations, respectively. PAR1, PAR2, PAR3 and PAR4 were sampled every 1,000 generations. The first $25 \%$ of the trees was discarded as burn-in. Convergence was assessed by determining the average standard deviation of split frequencies $(<0.01)$. The posterior probabilities for internal nodes were calculated from the posterior density of trees.

Selective pressure analysis. In order to determine whether positive selection is involved in the evolution of the PAR family, CodeML in PAML 4.7 was used with the models M0 (one ratio), M1a (near neutral), M2a (positive selection), M3 (discrete), M7 $(\beta)$ and M8 ( $\beta$ and $\omega)$ (23-25). M7 is a null model that does not allow for any codons with $\omega>1$, whereas the M8 model allows for positive selective sites $(\omega>1)$. When the M8 model fit the data significantly better (P-value $<0.05$ ) than the null model $(\mathrm{M} 7)$, the presence of positive selection sites was suggested. Conversely, results with a P-value $>0.05$ were regarded as lacking any positive selection sites. The non-synonymous/synonymous substitutions rate ratio $(\omega=\mathrm{dN} / \mathrm{dS})$ was also used to indicate selective pressure; $\omega>1$ indicates positive selection, $\omega<1$ indicates negative selection and $\omega=1$ indicates neutral evolution (26). Twice the difference in log likelihood between the M7 and M8 models (2 $\Delta \mathrm{l})$ was compared against $\chi^{2}$ with critical values of 0.01 significance levels. The number of non-synonymous substitutions $(\mathrm{dN})$ per non-synonymous site and the number of synonymous substitutions (dS) per synonymous site were computed by MEGA 5.1 using the modified Nei-Gojobori method. The transition/transversion ratio computed by MEGA 5.1 with the ML method was 1.4, 1.58, 1.72 and 1.16 corresponding to PAR1, PAR2, PAR3 and PAR3, respectively.

Functional divergence analysis. The multiple alignment of amino acid sequences among clusters of PAR1 and PAR2, PAR1 and PAR3, PAR1 and PAR4, PAR2 and PAR3, PAR2 and PAR4, and PAR3 and PAR4, were input into a Clustal format and type I functional divergence analysis was performed using Diverge (v2.0) with the maximum-likelihood estimation (MLE) and model-free method (MFE) $(27,28)$. The co-efficient of functional divergence $\theta(0<\theta<1)$ was used to indicate the functional divergence that occurred in different clusters of the PAR family. A significant result, i.e., $\theta>0$, suggests that it is desirable to identify the amino acid residues in the protein which have experienced a shift in their functional constraints. These sites may be relevant to the functional-structural differences between proteins.

Conservation of synteny analysis. To further study the evolution of the PAR gene family, we investigated the chromosomal distribution of PAR1, PAR2, PAR3 and PAR4 in eutheria, birds and teleosts. The location of chromosomes and the number of exons in the PAR family were searched in the Ensembl genome assemblies (http://www.ensembl.org/index.html).

\section{Results}

$P A R$ gene repertoires in vertebrates. To examine the origin and genetic evolution of the PAR family in vertebrates, we collected 57 vertebrate gene sequences from Ensembl and 
Table I. Number of functional and uncompleted genes in the vertebrate PAR family.

\begin{tabular}{|c|c|c|c|c|c|}
\hline Clade & Species & Name & $\begin{array}{l}\text { Functional } \\
\text { genes }\end{array}$ & $\begin{array}{l}\text { Uncompleted } \\
\text { genes }\end{array}$ & Total \\
\hline \multirow[t]{36}{*}{ Eutheria } & Homo sapiens & Human & 4 & & 4 \\
\hline & Otolemur garnettii & Bushbaby & 4 & & 4 \\
\hline & Pan troglodytes & Chimpanzee & 4 & & 4 \\
\hline & Nomascus leucogenys & Gibbon & 3 & 1 & 4 \\
\hline & Gorilla gorilla & Gorilla & 4 & & 4 \\
\hline & Pongo abelii & Orangutan & 4 & & 4 \\
\hline & Macaca mulatta & Macaque & 4 & & 4 \\
\hline & Callithrix jacchus & Marmoset & 4 & & 4 \\
\hline & Microcebus murinus & Mouse lemur & 1 & 2 & 3 \\
\hline & Tarsius syrichta & Tarsier & & 1 & 1 \\
\hline & Vicugna pacos & Alpaca & 1 & 2 & 3 \\
\hline & Bos taurus & Cow & 4 & & 4 \\
\hline & Felis catus & Cat & 4 & & 4 \\
\hline & Canis lupus familiaris & Dog & 4 & & 4 \\
\hline & Tursiops truncatus & Dolphin & 3 & 1 & 4 \\
\hline & Mustela putorius furo & Ferret & 4 & & 4 \\
\hline & Equus caballus & Horse & 3 & 1 & 4 \\
\hline & Pteropus vampyrus & Megabat & 3 & 1 & 4 \\
\hline & Myotis lucifugus & Microbat & 2 & 1 & 3 \\
\hline & Sus scrofa & Pig & 4 & & 4 \\
\hline & Ailuropoda melanoleuca & Panda & 3 & 1 & 4 \\
\hline & Sorex araneus & Shrew & 2 & & 2 \\
\hline & Loxodonta africana & Elephant & 3 & 1 & 4 \\
\hline & Procavia capensis & Hyrax & 1 & 3 & 4 \\
\hline & Macropus eugenii & Wallaby & 1 & 1 & 2 \\
\hline & Echinops telfairi & Lesser hedgehog tenrec & 1 & 1 & 2 \\
\hline & Choloepus hoffmanni & Sloth & 2 & 1 & 3 \\
\hline & Sarcophilus harrisii & Tasmanian devil & 4 & & 4 \\
\hline & Cacia porcellus & Guinea pig & 2 & 1 & 3 \\
\hline & Mus musculus & Mouse & 4 & & 4 \\
\hline & Oryctolagus cuniculus & Rabbit & 2 & 1 & 3 \\
\hline & Ochotona princeps & Pika & 2 & & 2 \\
\hline & Dipodomys ordii & Kangaroo rat & & 3 & 3 \\
\hline & Rattus norvegicus & Rat & 4 & & 4 \\
\hline & Ictidomys tridecemlineatus & Squirrel & 2 & & 2 \\
\hline & Tupaia belangeri & Tree shrew & & 2 & 2 \\
\hline Metatheria & Monodelphis domestica & Opossum & 5 & & 5 \\
\hline Prototheria & Ornithorhynchus anatinus & Platypus & 3 & & 3 \\
\hline \multirow[t]{5}{*}{ Birds } & Anas platyrhynchos & Duck & 4 & 1 & 5 \\
\hline & Gallus gallus & Chicken & 5 & & 5 \\
\hline & Ficedula albicollis & Flycatcher & 5 & & 5 \\
\hline & Meleagris gallopavo & Turkey & 1 & 4 & 5 \\
\hline & Taeniopygia guttata & Zebra finch & 3 & 1 & 4 \\
\hline \multirow[t]{3}{*}{ Reptiles } & Anolis carolinensis & Anole lizard & 2 & & 2 \\
\hline & Dasypus novemcinctus & Armadillo & 3 & & 3 \\
\hline & Pelodiscus sinensis & Chinese softshell turtle & 2 & 1 & 3 \\
\hline Amphibia & Xenopus tropicalis & Xenopus & 5 & & 5 \\
\hline
\end{tabular}


Table I. Continued.

\begin{tabular}{|c|c|c|c|c|c|}
\hline Clade & Species & Name & $\begin{array}{l}\text { Functional } \\
\text { genes }\end{array}$ & $\begin{array}{l}\text { Uncompleted } \\
\text { genes }\end{array}$ & Total \\
\hline \multirow[t]{9}{*}{ Teleost } & Latimeria chalumnae & Coelacanth & 7 & 9 & 16 \\
\hline & Xiphophorus maculatus & Platyfish & 6 & 1 & 7 \\
\hline & Oreochromis niloticus & Tilapia & 6 & 4 & 10 \\
\hline & Danio rerio & Zebrafish & 11 & 1 & 12 \\
\hline & Gadus morhua & Cod & & 6 & 6 \\
\hline & Takifugu rubripes & Fugu & 2 & 3 & 5 \\
\hline & Oryzias latipes & Medaka & 2 & 3 & 5 \\
\hline & Gasterosteus aculeatus & Stickleback & & 6 & 6 \\
\hline & Tetraodon nigroviridis & Tetraodon & & 4 & 4 \\
\hline Total & & 57 & 169 & 69 & 238 \\
\hline
\end{tabular}

PAR, protease-activated receptor.

tBLASTn (NCBI) using Homo sapiens genes as queries. Following the elimination of uncompleted sequences in Ensembl and NCBI, 169 functional gene sequences were applied to this study (Table I). The taxa comprised 18 non-mammals (9 teleost fish: coelacanth, platyfish, tilapia, zebrafish, cod, fugu, medaka, stickleback and tetraodon; 1 amphibian: xenopus; 3 reptiles: anole lizard, armadillo and Chinese softshell turtle; 5 birds: duck, chicken, flycatcher, turkey and zebra finch) and 38 mammals that include 10 primates (human, bushbaby, chimpanzee, gorilla, gibbon, orangutan, macaque, marmoset, mouse lemur and tarsier), 26 other mammals (alpaca, cow, cat, dog, dolphin, ferret, horse, megabat, microbat, pig, panda, shrew, elephant, hyrax, wallaby, lesser hedgehog tenrec, sloth, Tasmanian devil, guinea pig, mouse, rabbit, pika, rat, kangaroo rat, squirrel and tree shrew), 1 metatheria (opossum) and 1 prototheria (platypus). According to the completeness of genes, we divided the collected genes into 2 groups as follows: i) functional genes, which are sequences containing full-length open reading frames (ORFs) and ii) uncompleted genes, meaning those that lack start or stop codes or codes in the middle of sequences. Based on these criteria, we identified 169 functional genes and 69 uncompleted genes from a total of 238 PAR family genes. The number of PAR genes varied between the non-mammalian and mammalian vertebrates; 58 functional genes were identified in the non-mammalian species, ranging from 1 in the turkey to 11 in zebrafish; 49 uncompleted genes were found in the non-mammalian species, ranging from 1 in the duck, zebra finch, Chinese softshell turtle, platyfish and zebrafish to 9 in the coelacanth. A total of 111 functional genes were identified in mammals and these ranged from 1 in the mouse lemur, alpaca, hyrax, wallaby, and lesser hedgehog tenrec to 5 in the opossum.

Phylogenetic analysis of the PAR genes. To examine the evolutionary relationship of the PAR gene family, the 169 functional genes that were identified from all 238 genes were analyzed by the ML method and Bayesian inference. These yielded a similar result. The phylogenetic tree constructed by ML is shown in Fig. 1. Based on this model, the PAR gene family can be divided into 4 clades, PAR1, PAR2, PAR3 and PAR4, suggesting that PAR gene sequences have major differences. To further examine the phylogenetic relationship of each clade, we constructed 4 sub-trees for PAR1, PAR2, PAR3 and PAR4 (Figs. 2-5) using Bayesian inference. In the PAR1 clade, the posterior probability is $>58$. Teleost PAR1 genes form a group separate from eutherian and bird genes. We found that there is only 1 AR1 copy in eutheria, metatheria, bird, amphibian and reptile, whereas there are 6 copies in the zebrafish, 3 copies in the tilapia and 2 copies in the platyfish. In addition, the posterior probability of the teleost genes is higher than that of eutherian and bird genes. In the PAR2 clade, the posterior probability is $>54$. Primates, birds and teleost PAR2s form a group separate from other vertebrate genes. There is only 1 PAR 2 copy in most vertebrates apart from teleosts ( 2 copies in zebrafish, 2 copies in tilapia, 2 copies in platyfish and 2 copies in coelacanth). In the PAR3 clade, the posterior probability is $>54$, with birds and teleosts each forming a group separate from other species. There is only 1 PAR 3 copy in most vertebrates apart from zebrafish (2 copies). The posterior probability of primates is 100 and the posterior probabilities between megabat and microbat, cow and pig, zebra finch and flycatcher, tilapia and zebrafish, opossum and Tasmanian devil, rat and mouse are also 100. In the PAR4 clade, the posterior probability is $>57$, with birds and teleosts again forming a group separate from other species. Teleosts, amphibians and birds have multiple copies in the PAR4 clade (3 copies in coelacanth, 2 copies in xenopus, 2 copies in flycatcher and 2 copies in the chicken).

Functional divergence analysis. The 4 PAR clades were analyzed to determine whether there is functional divergence between them (PAR1 vs. PAR2, PAR1 vs. PAR3, PAR1 vs. PAR4, PAR2 vs. PAR3, PAR2 vs. PAR4 and PAR3 vs. PAR4). We estimated type I divergence using Diverge v2.0 (Table II). The results revealed that all $\theta$ values were significant $(>0)$, indicating that a site-specific rate shift during gene duplication was a common phenomenon in the evolution of the vertebrate PAR family. We also noticed that the 


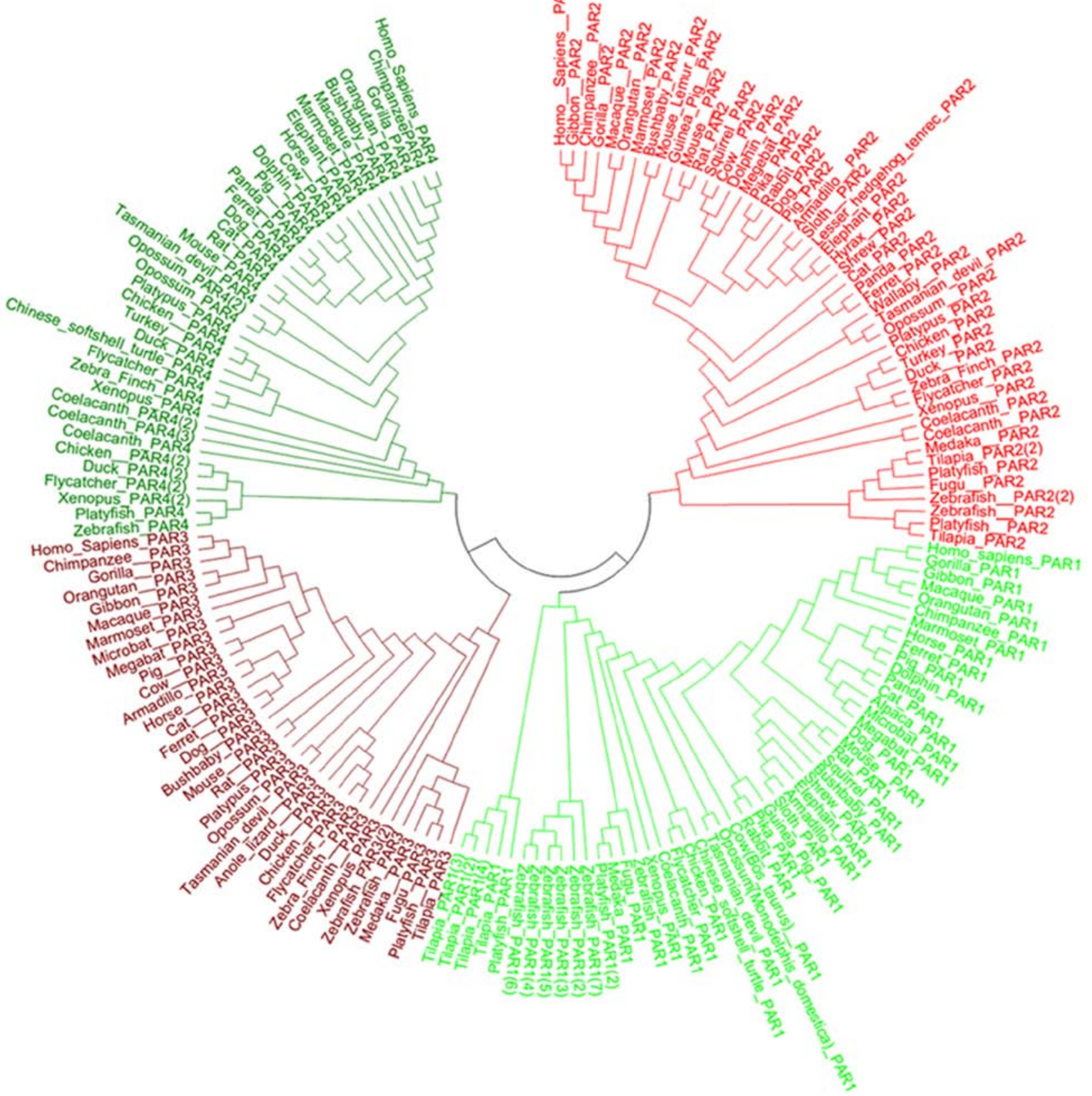

Figure 1. Maximum likelihood (ML) tree of the protease-activated receptor (PAR) gene family in vertebrates.

Table II. Functional divergence of the PAR family genes.

\begin{tabular}{lcccc}
\hline & \multicolumn{2}{c}{$\theta$ value } & & Standard error \\
\cline { 2 - 3 } \cline { 5 - 5 } \cline { 5 - 5 } & MFE method & MLE method & & MFE method \\
\hline PAR1 vs. PAR2 & 0.397 & 0.364 & \\
PAR1 vs. PAR3 & 0.370 & 0.395 & & 0.056 \\
PAR1 vs. PAR4 & 0.360 & 0.382 & & 0.057 \\
PAR2 vs. PAR3 & 0.433 & 0.494 & & 0.061 \\
PAR2 vs. PAR4 & 0.313 & 0.372 & 0.053 \\
PAR3 vs. PAR4 & 0.323 & 0.372 & 0.058 \\
\hline
\end{tabular}

PAR, protease-activated receptor; $\theta$, coefficient of functional divergence; MFE, model-free method; MLE, maximum-likelihood estimation.
$6 \theta$ values of PAR1 vs. PAR2, PAR1 vs. PAR3, PAR1 vs. PAR4, PAR2 vs. PAR3, PAR2 vs. PAR4 and PAR3 vs. PAR4 were all $<0.5$, suggesting that they did not diverge markedly.

Selective pressure analysis. To determine whether positive selection drove the evolution of the PAR gene family, we calculated the $\mathrm{dN}$ and $\mathrm{dS}$ distances between each pair of the sequences from the 4 clades (Fig. 6). The distance between $\mathrm{dN}$ and $\mathrm{dS}$ is large in the pairwise comparison of the PAR1 sequences, suggesting that positive selection is involved in PAR1 evolution. The value of $\mathrm{dN}$ is not significantly higher than the value of $\mathrm{dS}$ in the pairwise comparisons of the PAR2, PAR3 and PAR4 sequences. Most values of $\mathrm{dN} / \mathrm{dS}$ in these sequences were distributed below the diagonal, suggesting that positive selection is not involved in the evolution of these 
Table III. Site-specific tests for positive selection sites in the PAR gene family.

\begin{tabular}{|c|c|c|c|c|c|}
\hline & Models & InL & Estimates of parameters & $2 \Delta \mathrm{I}$ & Positive selection sites \\
\hline PAR1 & $\begin{array}{l}\operatorname{M7}(\beta) \\
\operatorname{M8}(\beta \text { and } \omega)\end{array}$ & $\begin{array}{l}-18520.05 \\
-18498.03\end{array}$ & $\begin{array}{l}\mathrm{p}=0.42638, \mathrm{q}=1.45124 \\
\mathrm{p} 0=0.95469, \mathrm{p}=0.54737, \mathrm{q}=2.73176 \\
(\mathrm{p} 1=0.04531) \mathrm{w}=1.26332\end{array}$ & $\begin{array}{l}22.02(\mathrm{P}<0.01) \\
22.02(\mathrm{P}<0.01)\end{array}$ & $\begin{array}{l}\text { NA } \\
65 \mathrm{G}^{* *} 76 \mathrm{~K}^{* *}\end{array}$ \\
\hline PAR2 & $\begin{array}{l}\operatorname{M7}(\beta) \\
\operatorname{M8}(\beta \text { and } \omega)\end{array}$ & $\begin{array}{l}-19806.32 \\
-19806.32\end{array}$ & $\begin{array}{l}\mathrm{p}=0.49569, \mathrm{q}=2.76102 \\
\mathrm{p} 0=0.99999, \mathrm{p}=0.49569, \mathrm{q}=2.76102 \\
(\mathrm{p} 1=0.00001) \mathrm{w}=2.89180\end{array}$ & $\begin{array}{l}0(\mathrm{P}=1.00) \\
0(\mathrm{P}=1.00)\end{array}$ & $\begin{array}{l}\text { NA } \\
\text { None }\end{array}$ \\
\hline PAR3 & $\begin{array}{l}\operatorname{M7}(\beta) \\
\operatorname{M8}(\beta \text { and } \omega)\end{array}$ & $\begin{array}{l}-13970.25 \\
-13970.25\end{array}$ & $\begin{array}{l}\mathrm{p}=0.70619, \mathrm{q}=3.15181 \\
\mathrm{p} 0=0.99999, \mathrm{p}=0.70622 \\
\mathrm{q}=3.15206(\mathrm{p} 1=0.00001), w=1.00000\end{array}$ & $\begin{array}{l}0(\mathrm{P}=1.00) \\
0(\mathrm{P}=1.00)\end{array}$ & $\begin{array}{l}\text { NA } \\
\text { None }\end{array}$ \\
\hline PAR4 & $\begin{array}{l}\operatorname{M7}(\beta) \\
\operatorname{M8}(\beta \text { and } \omega)\end{array}$ & $\begin{array}{l}-16496.19 \\
-16496.19\end{array}$ & $\begin{array}{l}\mathrm{p}=0.70253, \mathrm{q}=3.17231 \\
\mathrm{p} 0=0.99999, \mathrm{p}=0.70253, \mathrm{q}=3.17230 \\
(\mathrm{p} 1=0.00001), w=5.42283\end{array}$ & $\begin{array}{l}0(\mathrm{P}=1.00) \\
0(\mathrm{P}=1.00)\end{array}$ & $\begin{array}{l}\text { NA } \\
\text { None }\end{array}$ \\
\hline
\end{tabular}

PAR, protease-activated receptor; $\operatorname{lnL}$, the log-likelihood difference between the two models; $2 \Delta \mathrm{I}$, twice the log-likelihood difference between the 2 models; NA, not allowed; None, no positive selection sites identified.

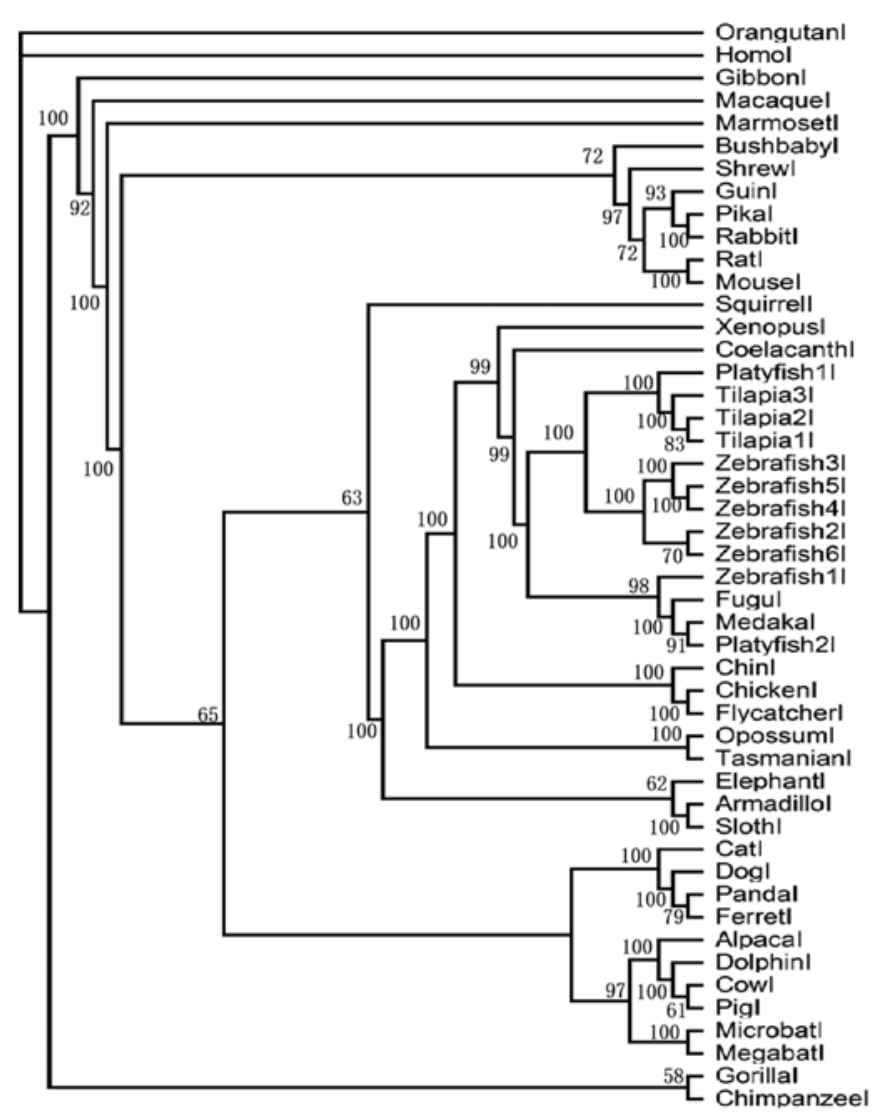

Figure 2. The sub-trees of protease-activated receptor (PAR1) were constructed using Bayesian inference.

3 clades. In addition, site-specific tests for positive selection were performed on the vertebrate PAR gene family using PAML4.7 (Table III) and some positive selection sites for PAR1 were found. The amino acids, $65 \mathrm{G}^{* *}$ and $76 \mathrm{~K}^{* *}$, were identified as positive selection sites (referring to Homo sapiens) in the PAR1 gene, whereas there were no positive selection sites found in the PAR2, PAR3 and PAR4 genes. The positive

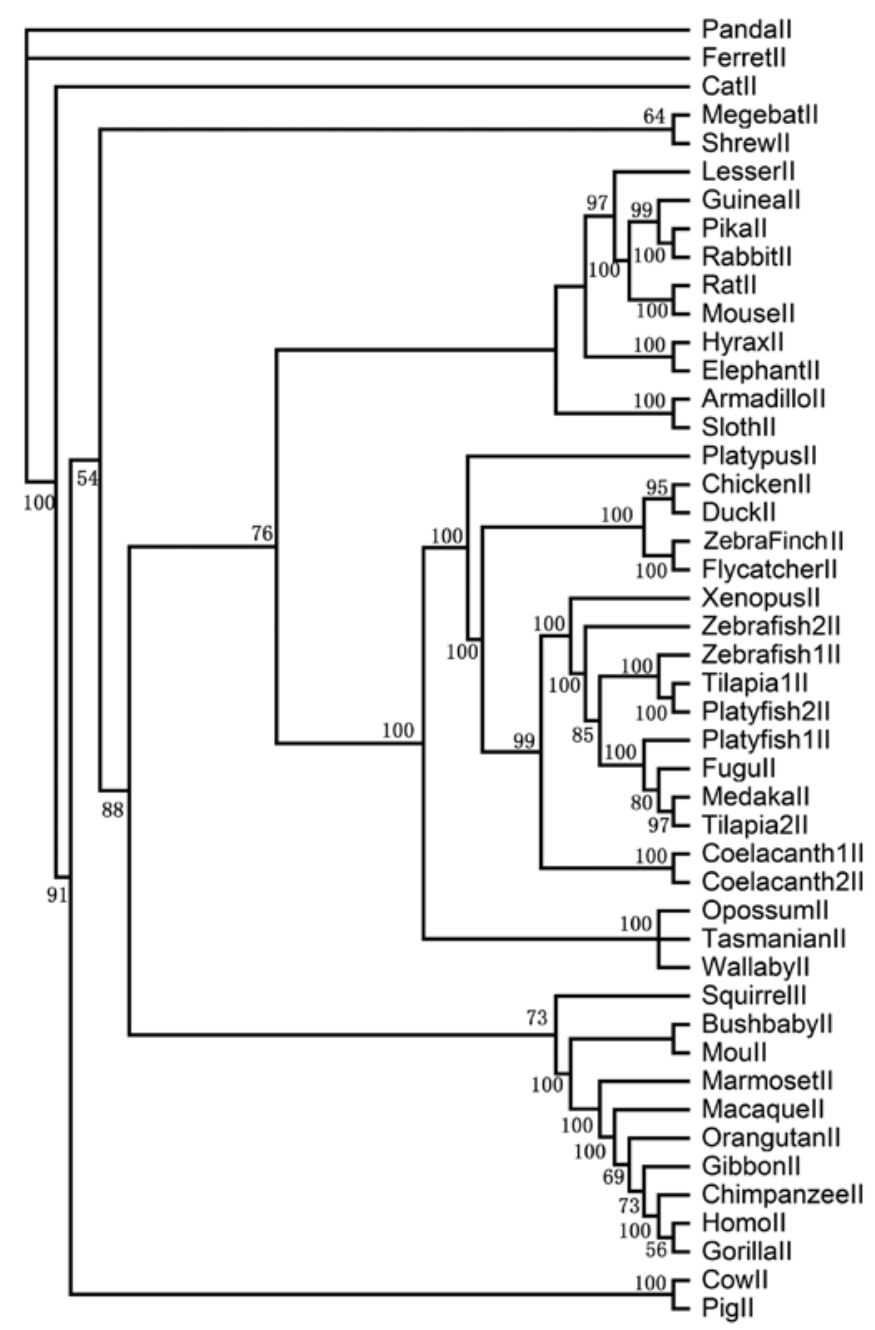

Figure 3. The sub-trees of protease-activated receptor (PAR2) were constructed using Bayesian inference.

selection sites identified by PAML 4.7 were the same as those identified by dN/dS analysis using MEGA 5.1. 


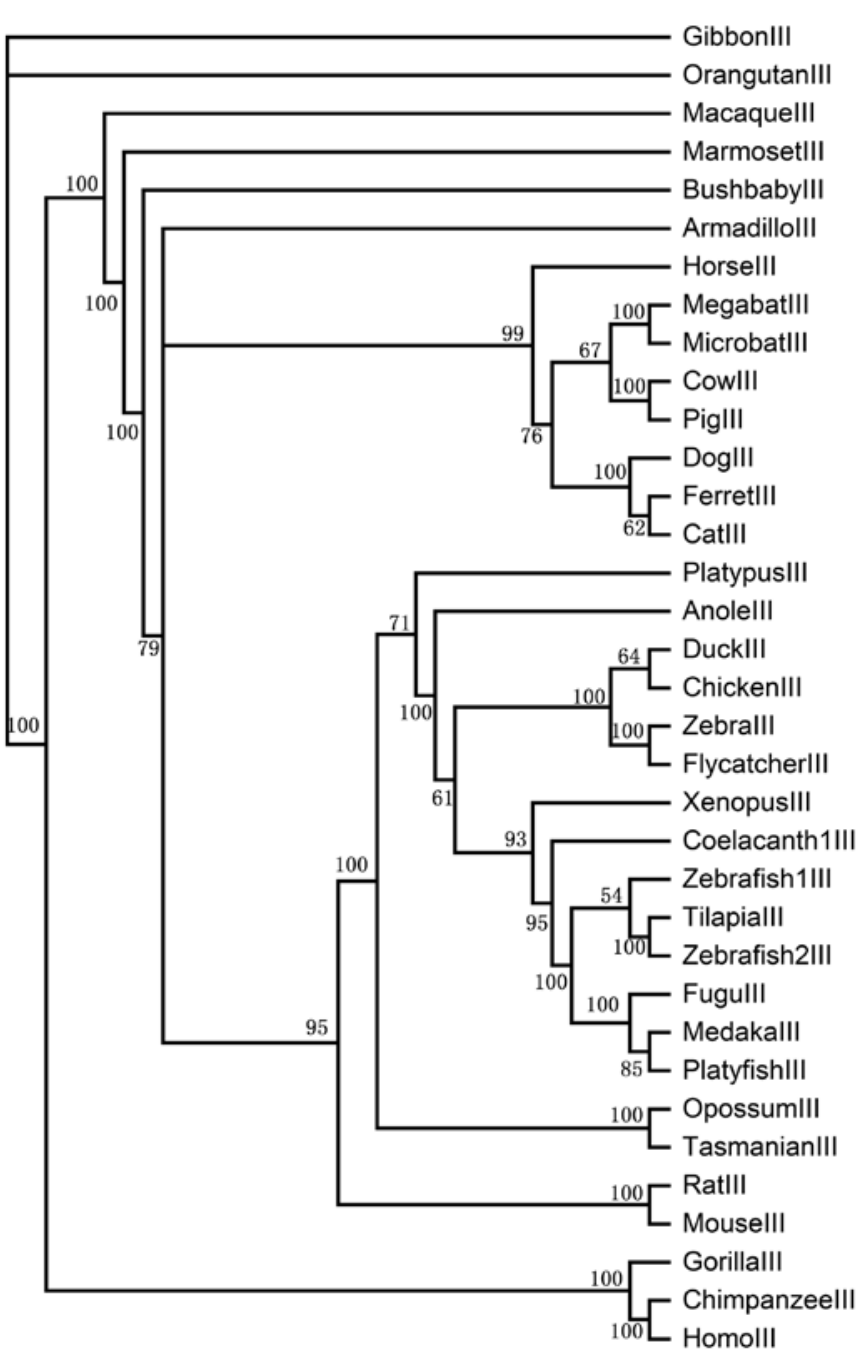

Figure 4. The sub-trees of protease-activated receptor (PAR3) were constructed using Bayesian inference.

Conservation of synteny in the PAR gene family. Based on the results shown in Fig. 7, we found that PAR1, PAR2, PAR3 are located on the same chromosome (F2R encoding PAR1, F2RL1 encoding PAR2 and F2RL2 encoding PAR3) and PAR4 is located on a different chromosome (F2RL3 encoding PAR4). For example, PAR1, PAR2 and PAR3 are located on chromosome 5 and PAR4 on chromosome 19 in humans, chimpanzees and orangutans. However, in teleosts, the chromosomal distribution of PARs differs from that observed in primates and birds. In this case, PAR1, PAR2 and PAR3 are not located on just 1 chromosome, but are distributed among different chromosomes. For example, in zebrafish, PAR1 is on chromosome 5, PAR2 is on chromosome 21 and PAR4 is on chromosome 14, while PAR3 is found on chromosomes 5 and 21. In the medaka, PAR1 is found on chromosome 9, PAR2 and PAR4 are found on chromosome 12 and PAR3 is found on chromosomes 9 and 12. Most of the PAR genes have 2 orthologous exons. However, there are exceptions in the PAR genes of many species (Table IV). The dog, dolphin, horse and macaque have 3 exons, while the marmoset and shrew have 4 exons in their PAR1 genes. The squirrel, ferret, megabat, medaka and lesser hedgehog have 3 exons, while the cat, wallaby and xenopus have 4 exons in their PAR2 genes. Megabat has three

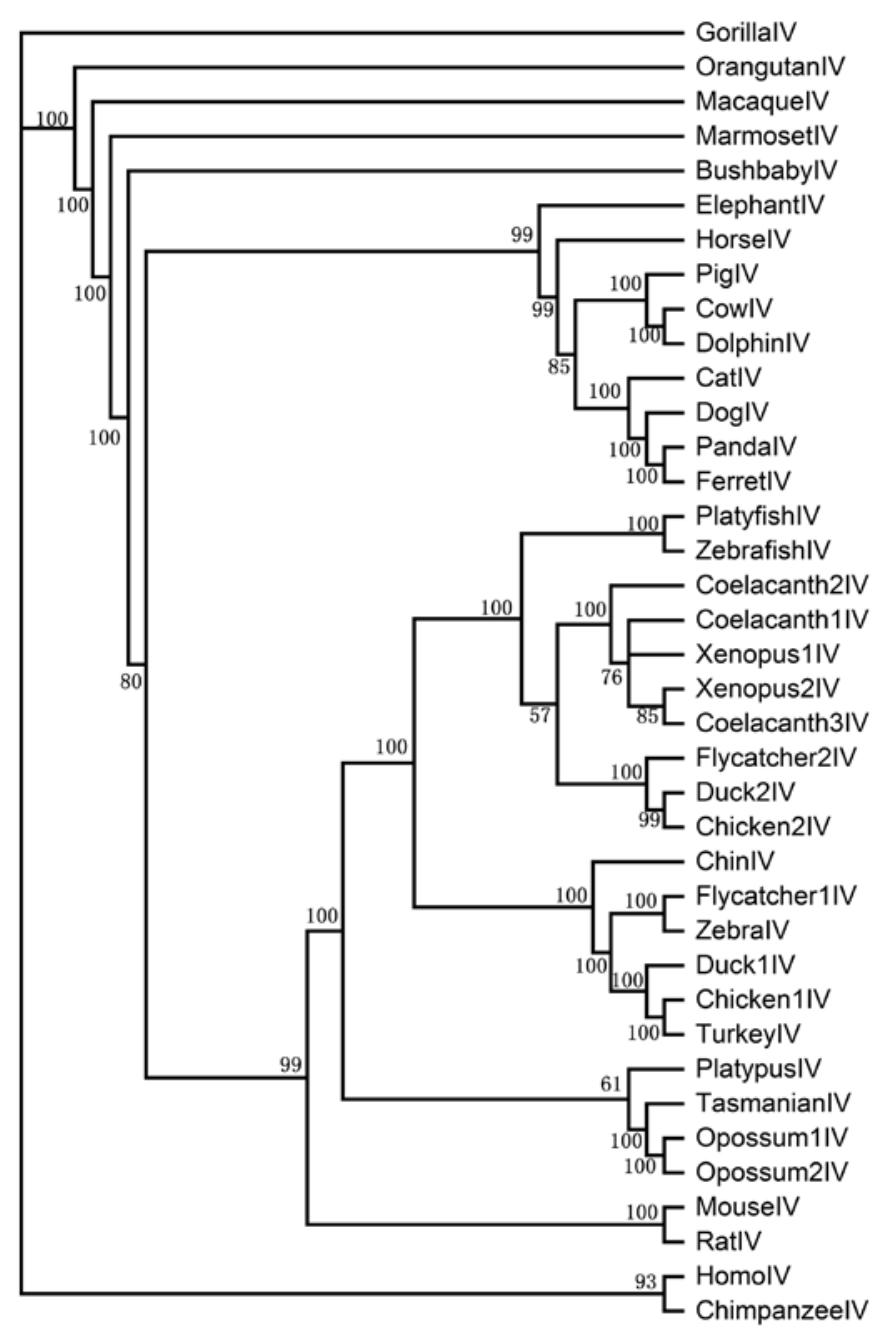

Figure 5. The sub-trees of protease-activated receptor (PAR4) were constructed using Bayesian inference.

exons in its PAR3 gene. The bushbaby and gibbon have 1 exon in their PAR4 genes, while the orangutan has 4 exons and the ferret has 3 exons in their PAR4 genes.

\section{Discussion}

The PAR family (PAR1-4) members belong to the GPCR family and are found in vertebrates. Based on an analysis of a number of different species, in the present study, we found that specific-specific gene duplication did not occur in the vertebrate PAR genes and that the lineage-specific gene expansion of PARs was observed only in teleosts, indicating that gene duplication occurred prior to the separation of these vertebrate species and the teleost cluster evolved following a birth-and-death model $(4,14,29)$.

The PAR genes were divided into 4 clades (PAR1, PAR2, PAR3 and PAR4) in the ML tree, suggesting that the PAR genes originated from 4 ancestors. In the ML tree, PAR1 and PAR2 are clustered into 1 subfamily and PAR3 and PAR4 are clustered into another subfamily, which suggested that the genes encoding PAR1 and PAR2 and the genes encoding PAR3 and PAR4 arose from a gene duplication event that was relatively recent in the history of the PAR family. In each of the 

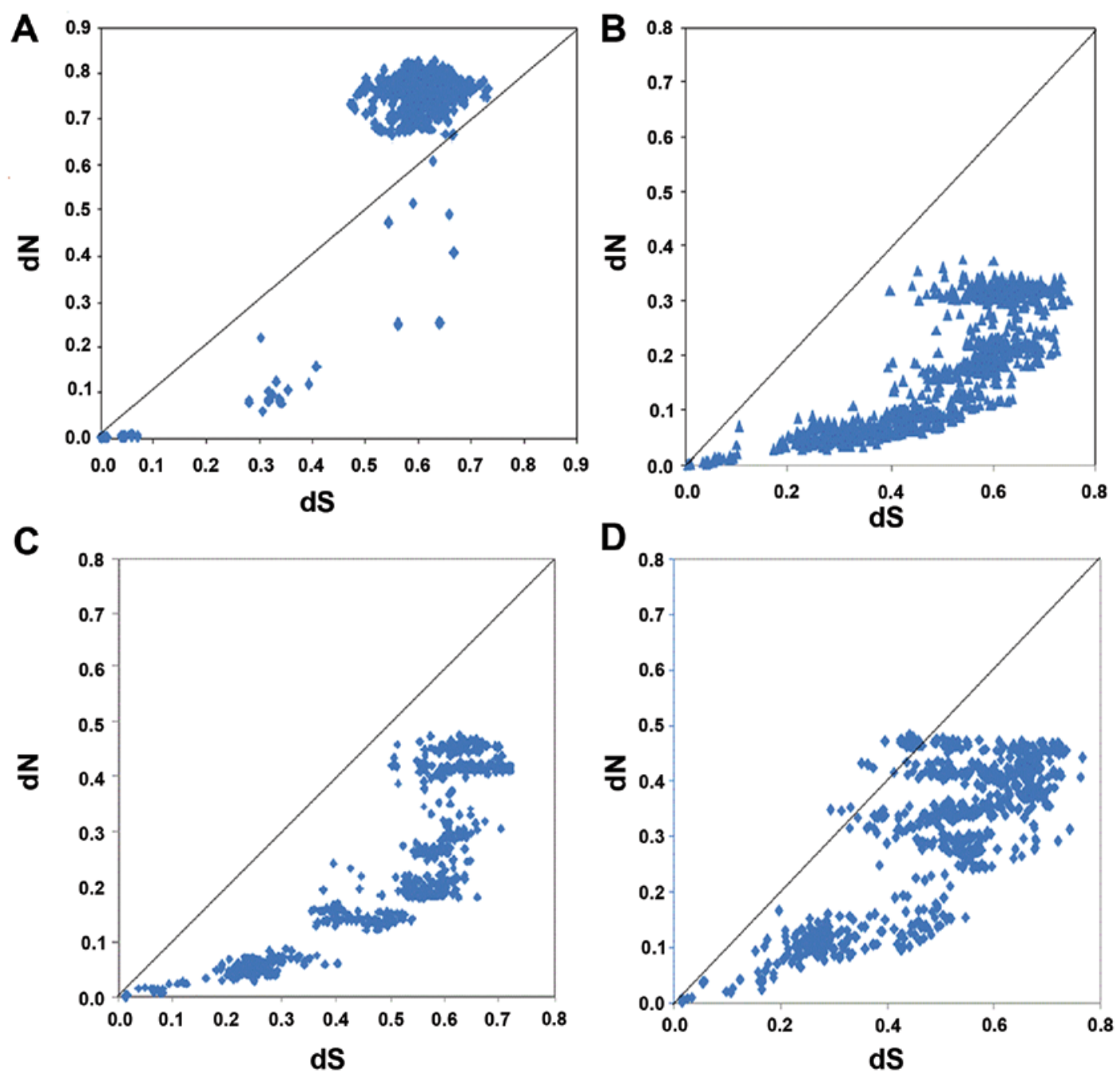

Figure 6. Pairwise comparisons of the number of non-synonymous substitutions $(\mathrm{dN})$ per non-synonymous site and the number of synonymous substitutions (dS) per synonymous site for the protease-activated receptor (PAR) gene family. Pairwise comparisons of dN and dS for (A) PAR1, (B) PAR2, (C) PAR3 and (D) PAR4.

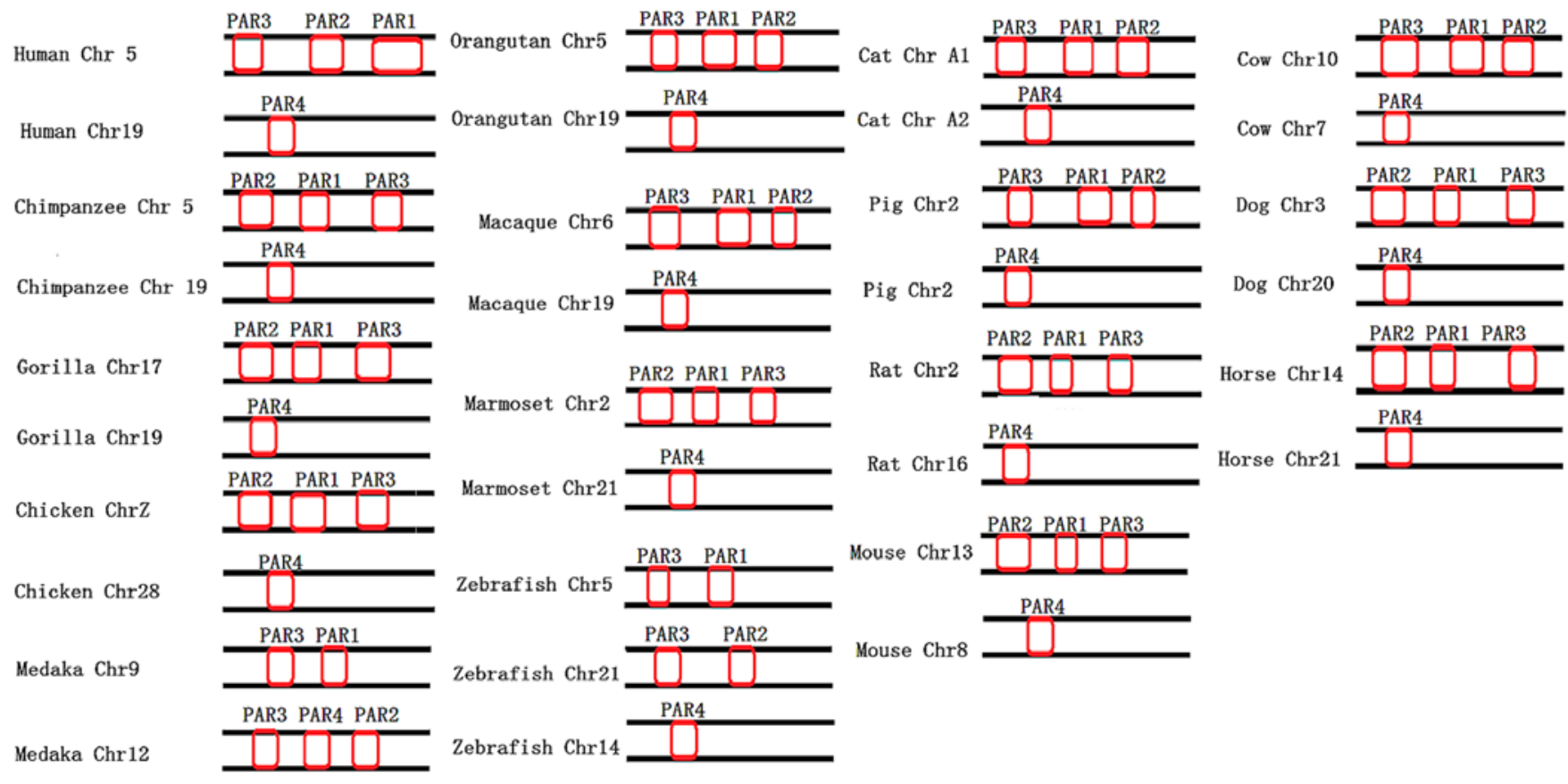

Figure 7. Chromosomal distribution of the protease-activated receptor (PAR) family. 
Table IV. The number of exons in the vertebrate PAR gene family.

No. of exons

\begin{tabular}{|c|c|c|c|c|}
\hline Species & in PAR1 & in PAR2 & in PAR3 & in PAR4 \\
\hline Human & 2 & 2 & 2 & 2 \\
\hline Bushbaby & 2 & 2 & 2 & 1 \\
\hline Chimpanzee & 2 & 2 & 2 & 2 \\
\hline Gibbon & 2 & 2 & & 1 \\
\hline Gorilla & 2 & 2 & 2 & 2 \\
\hline Orangutan & & 2 & 2 & 4 \\
\hline Macaque & 3 & 2 & 2 & 2 \\
\hline Marmoset & 4 & 2 & 2 & 2 \\
\hline Mouse lemur & & & 2 & \\
\hline Alpaca & 2 & 2 & & \\
\hline Cow & 2 & 2 & 2 & 2 \\
\hline Cat & 2 & 4 & 2 & \\
\hline Dog & 3 & 2 & & 2 \\
\hline Dolphin & 3 & 2 & & 2 \\
\hline Ferret & 2 & 3 & 2 & 3 \\
\hline Horse & 3 & 1 & 2 & \\
\hline Megabat & 2 & 3 & 3 & \\
\hline Pig & 2 & 2 & 2 & 2 \\
\hline Panda & & 3 & & 2 \\
\hline Shrew & 4 & & & \\
\hline Elephant & & 2 & & \\
\hline Hyrax & & 2 & & \\
\hline Wallaby & & 4 & & \\
\hline $\begin{array}{l}\text { Lesser hedgehog } \\
\text { tenrec }\end{array}$ & & 3 & & \\
\hline Sloth & 2 & 2 & & \\
\hline Tasmanian devil & 2 & 2 & 2 & 2 \\
\hline Mouse & 2 & 2 & 2 & 2 \\
\hline Rabbit & & 2 & & \\
\hline Rat & & 2 & 2 & 2 \\
\hline Squirrel & 2 & 3 & & \\
\hline Opossum & 2 & 2 & 2 & 2 \\
\hline Platypus & & 2 & 1 & \\
\hline Chicken & 2 & 2 & 2 & \\
\hline Flycatcher & 2 & & 2 & \\
\hline $\begin{array}{l}\text { Chinese softshell } \\
\text { turtle }\end{array}$ & 2 & 1 & & 2 \\
\hline Armadillo & 1 & & & \\
\hline Xenopus & & 4 & 2 & \\
\hline Coelacanth & 3 & 2 & & 2 \\
\hline Platyfish & & 2 & & 2 \\
\hline Tilapia & 2 & 2 & 2 & \\
\hline Zebrafish 1 & 2 & 2 & 2 & 2 \\
\hline Zebrafish 2 & 4 & & & \\
\hline Zebrafish 3 & 3 & & & \\
\hline Zebrafish 4 & 4 & & & \\
\hline Zebrafish 5 & 3 & & & \\
\hline Medaka & & 3 & & \\
\hline
\end{tabular}

PAR, protease-activated receptor.
4 PAR clades, teleosts, primates and other mammalian species are separated from the other vertebrates, forming independent clusters. In the process of evolution, only PAR1 was found to have arisen from positive selection, while no evidence for positive selection was found with PAR2, PAR3 and PAR4. The identified positive selection sites of PAR1 are $65 \mathrm{G}$ and $76 \mathrm{~K}$ (a signal peptide of approximately 20 amino acids in length is cleaved). These two amino acids are located on the $\mathrm{N}$-terminus of PAR1. The thrombin cleavage site on the N-terminus of PAR1 is located between Arg41 and Ser42, thus, the positive selection sites are still present on the new N-terminus created following the activation of PAR1. These positive selection sites may affect the cleavage of PAR1 or the subsequent binding of the novel $\mathrm{N}$-terminus with the body of the receptor. The results of positive selection analysis revealed that the PAR2, PAR3 and PAR4 lineages are highly conserved in vertebrates, while PAR1 is associated with environmental adaptation. The way in which PAR1 helps vertebrates adapt to their environment warrants further investigation. Based on the conservation of synteny analysis of the PAR gene family, we found that the PAR4 genes are located on a chromosome distinct from those of the genes encoding PAR1-3 in all vertebrates apart fromteleosts, in which the PARs genes are distributed on at least two chromosomes and PAR4 is not on a chromosome alone. This finding suggested that chromosomal fusion may have occurred during the evolution of teleosts. There are 2 exons in most of the vertebrate PARs, implying that they may be derived from the same ancestor and that exon rearrangement in PARs may not have occurred during the evolution of the PAR gene family in vertebrates. Functional divergences were found to have occurred between the PAR1, PAR2, PAR3 and PAR4 genes following analysis by the software, Diverge v2.0, implying that the PARs evolved individually in vertebrates. Different PARs have various functions.

Studies have demonstrated that PAR1 is involved in many functions, including the alteration of vascular tone and permeability, angiogenesis, and smooth muscle cell proliferation (30-32). As a receptor for serine proteases, following cleavage at a special site on the N-terminus of PAR2, activated PAR2 has been found to play a critical role in inflammation, immunity and angiogenesis (33). It has been demonstrated that PAR3 reduces the platelet response to thrombin, thereby providing protection from thrombosis, and mediates activated protein C anti-apoptotic signaling (34). PAR4 is involved in a number of functions, such as reducing platelet response to thrombin, providing protection from thrombosis in the cardiovascular system, regulating colonic nociception and inhibiting hypersensitivity in the nervous system $(35,36)$. Interaction also exists among the members of the PAR family and may affect the individual functions of the PARs. PAR3 can affect the signaling of PAR4 and strengthen the activation of PAR4. In a study on mouse platelets, PAR3 was activated at low thrombin concentrations, while PAR4 was activated at high thrombin concentrations (37). It has been demonstrated that PAR3 serves as a co-factor for the activation of PAR4 at low thrombin concentrations (38). In contrast to PAR3 co-factoring with PAR4, PAR1 modulates the activity of PAR2 through a different mechanism. Following cleavage by thrombin, the $\mathrm{N}$-terminus of PAR1 was found to unmask a tethered ligand domain, which binds in trans to activate PAR2 through an 
intermolecular liganding mechanism that elicits a special signaling response (39).

In conclusion, in this study, we examined the evolution of the PAR family by constructing phylogenetic trees, positive selection analysis, functional divergence analysis and conservation of synteny analysis. We concluded that the 4 members of the vertebrate PAR family originated from 4 ancestors and only PAR1 evolved by positive selection. In addition, in their separate evolution, there was no significant divergence of the functions of individual PARs. The evolutionary rates of the 4 PAR members were consistent. The findings of our study provide a theoretical background for the evolution of the PAR gene family in vertebrates.

\section{Acknowledgements}

This study was sponsored by the National Natural Science Foundation of China (nos. 31340073, 81373128, 81273274 and 30972822), the Great Project (no. 2011ZX08011-005) from the Major Program of National Science and Technology of China, the Special Research Project (no. 201300000159) from the Science and Information Technology of Guangzhou. The National Major Scientific and Technological Special Project for 'Significant New Drugs Development' (no. 2011ZX09302-003 -02), the Jiangsu Province Major Scientific and Technological Special Project (no. BM2011017) and a project funded by the Priority Academic Program Development of Jiangsu Higher Education Institutions.

\section{References}

1. Adams MN, Ramachandran R, Yau MK, Suen JY, Fairlie DP, Hollenberg MD and Hooper JD: Structure, function and pathophysiology of protease activated receptors. Pharmacol Ther 130 248-282, 2011

2. Ossovskaya VS and Bunnett NW: Protease-activated receptors: contribution to physiology and disease. Physiol Rev 84: 579-621, 2004.

3. Vergnolle N, Ferazzini M, D'Andrea MR, Buddenkotte J and Steinhoff M: Proteinase-activated receptors: novel signals for peripheral nerves. Trends Neurosci 26: 496-500, 2003.

4. Steinhoff M, Buddenkotte J, Shpacovitch V, Rattenholl A, Moormann C, Vergnolle N, Luger TA and Hollenberg MD: Proteinase-activated receptors: transducers of proteinase-mediated signaling in inflammation and immune response. Endocr Rev 26: $1-43,2005$.

5. Hamm HE: How activated receptors couple to $\mathrm{G}$ proteins. Proc Natl Acad Sci USA 98: 4819-4821, 2001.

6. De Candia E: Mechanisms of platelet activation by thrombin: a short history. Thromb Res 129: 250-256, 2012.

7. Kawabata A: Gastrointestinal functions of proteinase-activated receptors. Life Sci 74: 247-254, 2003.

8. Schmidlin F and Bunnett NW: Protease-activated receptors: how proteases signal to cells. Curr Opin Pharmacol 1: 575-582, 2001.

9. Yau MK,Liu Land Fairlie DP: Toward drugs for protease-activated receptor 2 (PAR2). J Med Chem 56: 7477-7497, 2013.

10. Ramachandran R, Noorbakhsh F, Defea K and Hollenberg MD: Targeting proteinase-activated receptors: therapeutic potential and challenges. Nat Rev Drug Discov 11: 69-86, 2012.

11. Böhm SK, Grady EF and Bunnett NW: Regulatory mechanisms that modulate signalling by G-protein-coupled receptors. Biochem J 322: 1-18, 1997.

12. Ishihara H, Connolly AJ, Zeng D, Kahn ML, Zheng YW, Timmons C, Tram T and Coughlin SR: Protease-activated receptor 3 is a second thrombin receptor in humans. Nature 386: 502-506, 1997.

13. Xu WF, Andersen H, Whitmore TE, Presnell SR, Yee DP, Ching A, Gilbert T, Davie EW and Foster DC: Cloning and characterization of human protease-activated receptor 4 . Proc Natl Acad Sci USA 95: 6642-6646, 1998.
14. Gieseler F, Ungefroren H, Settmacher U, Hollenberg MD and Kaufmann R: Proteinase-activated receptors (PARs) - focus on receptor-receptor-interactions and their physiological and pathophysiological impact. Cell Commun Signal 11: 86, 2013.

15. Leger AJ, Covic L and Kuliopulos A: Protease-activated receptors in cardiovascular diseases. Circulation 114: 1070-1077, 2006.

16. Cirino $\mathrm{G}$ and Vergnolle N: Proteinase-activated receptors (PARs): crossroads between innate immunity and coagulation. Curr Opin Pharmacol 6: 428-434, 2006.

17. Vergnolle N: Protease-activated receptors as drug targets in inflammation and pain. Pharmacol Ther 123: 292-309, 2009.

18. Lee $\mathrm{H}$ and Hamilton JR: Physiology, pharmacology, and therapeutic potential of protease-activated receptors in vascular disease. Pharmacol Ther 134: 246-259, 2012.

19. Xu H, Echemendia N, Chen S and Lin F: Identification and expression patterns of members of the protease-activated receptor (PAR) gene family during zebrafish development. Dev Dyn 240: 278-287, 2011

20. Kahn ML, Hammes SR, Botka C and Coughlin SR: Gene and locus structure and chromosomal localization of the protease-activated receptor gene family. J Biol Chem 273: 23290-23296, 1998.

21. Tamura K, Peterson D, Peterson N, Stecher G, Nei M and Kumar S: MEGA5: molecular evolutionary genetics analysis using maximum likelihood, evolutionary distance, and maximum parsimony methods. Mol Biol Evol 28: 2731-2739, 2011.

22. Ronquist F and Huelsenbeck JP: MrBayes 3: Bayesian phylogenetic inference under mixed models. Bioinformatics 19: 1572-1574, 2003.

23. Posada D: jModelTest: Phylogenetic model averaging. Mol Biol Evol 25: 1253-1256, 2008.

24. Guindon S and Gascuel O: A simple, fast, and accurate algorithm to estimate large phylogenies by maximum likelihood. Syst Biol 52: 696-704, 2003.

25. Yang Z: PAML 4: Phylogenetic analysis by maximum likelihood. Mol Biol Evol 24: 1586-1591, 2007.

26. Yang Z, Nielsen R, Goldman $\mathrm{N}$ and Pedersen AM: Codon-substitution models for heterogeneous selection pressure at amino acid sites. Genetics 155: 431-449, 2000.

27. Gu X and Vander Velden K: DIVERGE: phylogeny-based analysis for functional-structural divergence of a protein family. Bioinformatics 18: 500-501, 2002.

28. Gu X: Statistical methods for testing functional divergence after gene duplication. Mol Biol Evol 16: 1664-1674, 1999.

29. Piontkivska $\mathrm{H}$ and Nei M: Birth-and-death evolution in primate MHC class I genes: divergence time estimates. Mol Biol Evol 20: 601-609, 2003.

30. Hirano K: The roles of proteinase-activated receptors in the vascular physiology and pathophysiology. Arterioscler Thromb Vasc Biol 27: 27-36, 2007.

31. Weiss EJ, Hamilton JR, Lease KE and Coughlin SR: Protection against thrombosis in mice lacking PAR3. Blood 100: 3240-3244, 2002.

32. Yue R, Li H, Liu H, Li Y, Wei B, Gao G, Jin Y, Liu T, Wei L, Du J and Pei G: Thrombin receptor regulates hematopoiesis and endothelial-to-hematopoietic transition. Dev Cell 22: 1092-1100, 2012.

33. Guo H, Liu D, Gelbard H, Cheng T, Insalaco R, Fernández JA, Griffin JH and Zlokovic BV: Activated protein C prevents neuronal apoptosis via protease activated receptors 1 and 3 . Neuron 41: 563-572, 2004.

34. Busso N, Chobaz-Péclat V, Hamilton J, Spee P, Wagtmann N and So A: Essential role of platelet activation via protease activated receptor 4 in tissue factor-initiated inflammation. Arthritis Res Ther 10: R42, 2008.

35. Augé C, Balz-Hara D, Steinhoff M, Vergnolle $\mathrm{N}$ and Cenac $\mathrm{N}$ : Protease-activated receptor-4 (PAR 4): a role as inhibitor of visceral pain and hypersensitivity. Neurogastroenterol Motil 21: 1189-e107, 2009.

36. Coughlin SR: Thrombin signalling and protease-activated receptors. Nature 407: 258-264, 2000.

37. Lin H, Liu AP, Smith TH and Trejo J: Cofactoring and dimerization of proteinase-activated receptors. Pharmacol Rev 65: 1198-1213, 2013.

38. Rothmeier AS and Ruf W: Protease-activated receptor 2 signaling in inflammation. Semin Immunopathol 34: 133-149, 2012.

39. Hirano $\mathrm{K}$ and Kanaide $\mathrm{H}$ : Role of protease-activated receptors in the vascular system. J Atheroscler Thromb 10: 211-225, 2003. 